\title{
Development of 44 Novel Polymorphic SSR Markers for Determination of Shiitake Mushroom (Lentinula edodes) Cultivars
}

\author{
Hwa-Yong Lee ${ }^{1,2,+}$, Suyun Moon ${ }^{2,+}$, Donghwan Shim ${ }^{3,+}$, Chang Pyo Hong ${ }^{4}$, Yi Lee ${ }^{5}$, \\ Chang-Duck Koo ${ }^{1, *}$, Jong-Wook Chung ${ }^{5, *}$ and Hojin Ryu ${ }^{2, *}$ \\ 1 Department of Forest Science, Chungbuk National University, Cheongju 28644, Korea; \\ hoasis82@chungbuk.ac.kr \\ 2 Department of Biology, Chungbuk National University, Cheongju 28644, Korea; sooym21@naver.com \\ 3 Department of Forest Genetic Resources, National Institute of Forest Science, Suwon 16631, Korea; \\ shim104@korea.kr \\ 4 Theragen Etex Bio Institute, Suwon 16229, Korea; changpyo.hong@theragenetex.com \\ 5 Department of Industrial Plant Science and Technology, Chungbuk National University, \\ Cheongju 28644, Korea; leeyi22@chungbuk.ac.kr \\ * Correspondence: koocdm@chungbuk.ac.kr (C.-D.K.); jwchung73@chungbuk.ac.kr (J.-W.C.); \\ hjryu96@gmail.com (H.R.); Tel.: +82-43-261-2293 (H.R.) \\ + These authors contributed equally to this work.
}

Academic Editor: Paolo Cinelli

Received: 6 January 2017; Accepted: 21 March 2017; Published: 24 March 2017

\begin{abstract}
The shiitake mushroom (Lentinula edodes) is one of the most popular edible mushrooms in the world and has attracted attention for its value in medicinal and pharmacological uses. With recent advanced research and techniques, the agricultural cultivation of the shiitake mushroom has been greatly increased, especially in East Asia. Additionally, demand for the development of new cultivars with good agricultural traits has been greatly enhanced, but the development processes are complicated and more challenging than for other edible mushrooms. In this study, we developed 44 novel polymorphic simple sequence repeat (SSR) markers for the determination of shiitake mushroom cultivars based on a whole genome sequencing database of L. edodes. These markers were found to be polymorphic and reliable when screened in 23 shiitake mushroom cultivars. For the 44 SSR markers developed in this study, the major allele frequency ranged from 0.13 to 0.94 ; the number of genotypes and number of alleles were each 2-11; the observed and expected heterozygosity were $0.00-1.00$ and $0.10-0.90$, respectively; and the polymorphic information content value ranged from 0.10 to 0.89 . These new markers can be used for molecular breeding, the determination of cultivars, and other applications.
\end{abstract}

Keywords: shiitake mushroom; simple sequence repeat (SSR) marker; whole genome sequencing

\section{Introduction}

The shiitake mushroom (Lentinula edodes) belongs to genus Lentinula, family Omphalotaceae, order Agaricales [1], and is a type of white rot fungi. This mushroom is commonly cultivated in Asian countries such as Korea, China, Japan, Taiwan, and others [2,3]. It constitutes approximately $17 \%$ of the global mushroom supply and is one of the most popular edible mushrooms in the world [4]. In addition to its value as a food, the shiitake mushroom is useful for pharmacological components such as lentinan, which shows antitumour activity $[5,6]$.

To develop new mushroom cultivars, cross breeding, mutation breeding, transgenic breeding, and other approaches have been used [7]. The cultivar development of the shiitake mushroom 
is very difficult because the cultivation period is much longer than for the oyster mushroom (Pleurotus ostreatus), king oyster mushroom (P. eryngii), and winter mushroom (Flammulina velutipes) [8]. The traditional breeding for shiitake mushroom requires a lot of time and labor from strain selection for cultivation and identification of traits. Analysis of the genetic relationship between the relevant strains and the association of DNA markers in mushrooms can effectively increase breeding efficiency [9], because molecular markers save time in the selection process of the strain. During the last few decades, studies on the genetic diversity and population genetics in shiitake mushrooms have been conducted using various types of molecular markers, including restriction fragment length polymorphism (RFLP) [10], random amplified polymorphic DNA (RAPD) [11-13], amplified fragment length polymorphism (AFLP) [14], inter-simple sequence repeat (ISSR) $[12,15,16]$, sequence-characterized amplified region (SCAR) $[13,17,18]$, and sequence-related amplified polymorphism (SRAP) $[12,16]$. In spite of their diverse applications, the use of developed markers for the breeding and classification of shiitake mushrooms has been challengeable due to few available markers and little information regarding effectiveness for determination and specificity. Also, despite the advantages of simple sequence repeat (SSR) markers, such as co-dominant, highly polymorphic, reproducible, reliable, and distributed throughout the genome [19], the number of SSR markers available for Shiitake mushrooms are still scarce, with only a few expressed sequence tag-simple sequence repeat (EST-SSR) markers having been reported [20]. Thus, more reliable molecular markers are needed to enhance genetic analyses of the shiitake mushroom.

The traditional development of SSR markers was an experimentally long, labor-intensive, and economically costly process [21]. However, Next Generation Sequencing (NGS) technology is a powerful tool to find a large number of microsatellite loci through cost-effective and rapid identification [22]. In many recent studies, NGS-based transcriptome or genome sequencing is demonstrated to be efficient for the large-scale discovery of SSR loci in plants [21]. We have recently reported the genome sequence information of L. edodes [23], and have here developed higher polymorphic SSR markers based on the whole genome sequencing and determination of shiitake mushroom varieties. New SSR markers might be valuable tools to evaluate genetic variability and breeding in the shiitake mushroom.

\section{Materials and Methods}

\subsection{Fungi Materials}

To develop useful SSR markers in L. edodes, we selected the representative shiitake mushroom strains, which were successfully cultivated and distributed in the market in South Korea. Five strains from the National Institute of Forest Science in Korea Forest Service (http://www.forest.go.kr) and 18 strains from the Forest Mushroom Research Center (https://www.fmrc.or.kr/) were kindly provided. The list of strains are shown in Table 1. The mycelia of the strains were cultured for 10 days at $25^{\circ} \mathrm{C}$ in darkness.

\subsection{DNA Preparationument}

For DNA extraction, the cultured mycelia were frozen in liquid nitrogen and ground into powders. DNA extraction was performed using a GenEX Plant Kit (Geneall, Seoul, Korea) following the manufacturer's instructions. The extracted DNA was stored at $-80^{\circ} \mathrm{C}$. 
Table 1. List of shiitake mushroom (Lentinula edodes) strains.

\begin{tabular}{lll}
\hline No. & Strain Name & Cultivar Name \\
\hline 1 & KFRI 623 & Baekhwahyang \\
2 & KFRI 174 & Soohyangko \\
3 & KFRI 551 & Poongnyunko \\
4 & KFRI 2924 & Sanmaru $1 \mathrm{~h}_{\mathrm{O}}$ \\
5 & KFRI 2925 & Sanmaru $2 \mathrm{~h}_{\mathrm{O}}$ \\
6 & SJ101 & Sanjo $101 \mathrm{~h}_{\mathrm{O}}$ \\
7 & SJ102 & Sanjo $102 \mathrm{~h}_{\mathrm{O}}$ \\
8 & SJ103 & Sanjo $103 \mathrm{~h}_{\mathrm{O}}$ \\
9 & SJ108 & Sanjo $108 \mathrm{~h}_{\mathrm{O}}$ \\
10 & SJ109 & Sanjo $109 \mathrm{~h}_{\mathrm{O}}$ \\
11 & SJ110 & Sanjo $110 \mathrm{~h}_{\mathrm{O}}$ \\
12 & SJ111 & Sanjo $111 \mathrm{~h}_{\mathrm{O}}$ \\
13 & SJ301 & Sanjo $301 \mathrm{~h}_{\mathrm{O}}$ \\
14 & SJ501 & Sanjo $501 \mathrm{~h}_{\mathrm{O}}$ \\
15 & SJ702 & Sanjo 702 $\mathrm{h}_{\mathrm{O}}$ \\
16 & SJ704 & Sanjo 704 $\mathrm{h}_{\mathrm{O}}$ \\
17 & SJ705 & Sanjo 705 $\mathrm{h}_{\mathrm{O}}$ \\
18 & SJ706 & Sanjo 706 $\mathrm{h}_{\mathrm{O}}$ \\
19 & SJ707 & Sanjo 707 $\mathrm{h}_{\mathrm{O}}$ \\
20 & SJ708 & Sanjo 708 $\mathrm{h}_{\mathrm{O}}$ \\
21 & SJ709 & Sanjo 709 $\mathrm{h}_{\mathrm{O}}$ \\
22 & SJ710 & Sanjo 710 $\mathrm{h}_{\mathrm{O}}$ \\
23 & SJCAR & Chamaram \\
\hline
\end{tabular}

No. 1 5: National Institute of Forest Science in Korea Forest Service; No. 6 23: Forest Mushroom Research Center.

\subsection{Discovery of SSR Markers}

Over 1000 SSR loci of the shiitake mushroom were found in whole genome sequencing performed by Shim et al. [23] using L. edodes monokaryon strain B17 and comparing the resequencing data of 1 strain, Chamaram. We chose 205 SSR loci to test for polymorphism among the shiitake mushroom strains, and 44 SSR markers were finally selected for proper PCR conditions fixed in 23 strains (Supplementary Materials Figure S1).

The primer design parameters were set as follows: length range, 18-23 nucleotides with 21 as the optimum; PCR product size range, $150-200 \mathrm{bp}$; optimum annealing temperature (Ta), $58^{\circ} \mathrm{C}$; and GC content $50 \%-61 \%$, with $51 \%$ as the optimum.

The extracted DNA for PCR templates was diluted to $20 \mathrm{ng} / \mu \mathrm{L}$ after checking concentration using a K5600 micro spectrophotometer (DaAn Gene, Guangzhou, China). The PCR reaction mixture consisted of $2 \mu \mathrm{L}$ template DNA, $1 \mu \mathrm{L}$ each of forward and reverse primer ( 5 pmol), $10 \mu \mathrm{L} 2 \times \mathrm{i}$-Taq Master Mix (Intron biotechnology, Seongnam, Korea), and $6 \mu \mathrm{L}$ distilled water. PCR reactions were performed as follows: $95^{\circ} \mathrm{C}$ for $3 \mathrm{~min} ; 35$ cycles of $95^{\circ} \mathrm{C}$ for $30 \mathrm{~s}, 58^{\circ} \mathrm{C}$ for $30 \mathrm{~s}$, and $72{ }^{\circ} \mathrm{C}$ for $30 \mathrm{~s}$; and finally, $72{ }^{\circ} \mathrm{C}$ for $20 \mathrm{~min}$. The size of the PCR product was confirmed by fragment analyzer (Advanced Analytical Technologies, Ankeny, IA, USA).

The amplified SSR loci were scored for 23 shiitake mushroom strains. Major allele frequency $\left(\mathrm{M}_{\mathrm{AF}}\right)$, number of genotypes $\left(\mathrm{N}_{\mathrm{G}}\right)$, number of alleles $\left(\mathrm{N}_{\mathrm{A}}\right)$, observed heterozygosity $\left(\mathrm{H}_{\mathrm{O}}\right)$, expected heterozygosity $\left(\mathrm{H}_{\mathrm{E}}\right)$, and polymorphic information content (PIC) values were calculated by using PowerMarker V3.25 [24].

\section{Results and Discussion}

The 44 SSR markers consist of di-, tri-, tetra-, and pentanucleotide DNA motifs. The SSR motifs used include $59.09 \%$ dinucleotide repeats, $31.82 \%$ trinucleotide repeats, $6.82 \%$ tetranucleotide repeats, and $2.27 \%$ pentanucleotide repeats. The SSR motifs are AG/GA, CT/TC, AT/TA, AC/CA, CG/GC, and TG dinucleotide repeats; AGG/AGA/GGA, CAG/CGA/GCA, AGA, GAT, GCT, GTT, TCA, and TCG trinucleotide repeats; TACT/TATC, and CTTT tetranucleotide repeats; and CTTCC pentanucleotide repeats (Table 2). 
Table 2. Characteristics of the 44 simple sequence repeat (SSR) markers for shiitake mushroom (Lentinula edodes). Ta, annealing temperature.

\begin{tabular}{|c|c|c|c|c|c|c|}
\hline Marker & Primer Sequences $\left(5^{\prime}-3^{\prime}\right)$ & Expected Size & Motif & GenBank Accession No. & $\mathrm{Ta}\left({ }^{\circ} \mathrm{C}\right)$ & Description \\
\hline RL-LE-017 & $\begin{array}{l}\text { F: GTGCACTGTGCGATTGTTC } \\
\text { R: CAGCAAGGATGACTCTTGGA }\end{array}$ & 199 & CA & NM-0418-000001 & 59 & Subtilase family, Pro-kumamolisin, activation domain \\
\hline RL-LE-018 & $\begin{array}{l}\text { F: CCCACAGGTTTACAGAGTTCCT } \\
\text { R: GTGGACATCCACCTTTTGTC }\end{array}$ & 152 & $\mathrm{TA}$ & NM-0418-000002 & 59 & - \\
\hline RL-LE-019 & $\begin{array}{l}\text { F: TACTTTCGAAGCCAGCCA } \\
\text { R: GTAGCTCTTTAGGTCTGCTTGG }\end{array}$ & 191 & СТTCC & NM-0418-000003 & 58 & - \\
\hline RL-LE-020 & $\begin{array}{l}\text { F: GACGGAGTTGTCAAGATCTACC } \\
\text { R: ACCTAGGCTTTGCTCTACACAG }\end{array}$ & 173 & $\mathrm{AT}$ & NM-0418-000004 & 58 & - \\
\hline RL-LE-021 & $\begin{array}{l}\text { F: GCTTGAAGAGCGAGTTTGAG } \\
\text { R: CAAGACACGCTTCGTAGTCA }\end{array}$ & 200 & AG & NM-0418-000005 & 58 & Uso1/p115-like vesicle tethering protein, head region \\
\hline RL-LE-022 & $\begin{array}{l}\text { F: CAAACGAAGGAGGAGGTAGTTC } \\
\text { R: GAGTCCATTACTCATCGTGCTG }\end{array}$ & 199 & GCA & NM-0418-000006 & 60 & - \\
\hline RL-LE-023 & $\begin{array}{l}\text { F: GAGGTAGCACCAGTTGAGGTAA } \\
\text { R: ATAAGACTTCGTCTCGTCCTGC }\end{array}$ & 150 & AGA & NM-0418-000007 & 59 & - \\
\hline RL-LE-024 & $\begin{array}{l}\text { F: GTAAGGCTTTAGGACTCGTCG } \\
\text { R: CCACAGATGTTTCCGAGTTG }\end{array}$ & 187 & $\mathrm{TC}$ & NM-0418-000008 & 59 & - \\
\hline RL-LE-025 & $\begin{array}{l}\text { F: TTGGGAGATGCGAGTAGTTC } \\
\text { R: ATTCAGTCGCTCAGTAGGAGAC }\end{array}$ & 200 & AT & NM-0418-000009 & 58 & PCI domain, 26S proteasome subunit RPN7 \\
\hline RL-LE-026 & $\begin{array}{l}\text { F: GATTTGACGCTCACATCCC } \\
\text { R: CCCCTAAGTATGAGCTTCCGTA }\end{array}$ & 197 & AG & NM-0418-000010 & 59 & - \\
\hline RL-LE-027 & $\begin{array}{l}\text { F: GGGTCACAAGAGCAATGTAGAC } \\
\text { R: CTGTATGGTGATCAAGGACGAG }\end{array}$ & 192 & CT & NM-0418-000011 & 59 & - \\
\hline RL-LE-028 & $\begin{array}{l}\text { F: GAGACGACACGAGGAATTTG } \\
\text { R: GTCGTTCTCATTGGAGACTCTG }\end{array}$ & 174 & $\mathrm{CA}$ & NM-0418-000012 & 59 & Ras family \\
\hline RL-LE-029 & $\begin{array}{l}\text { F: CAAGATCCGTCGCCATATAC } \\
\text { R: AACTCACCCTCGTCTACCTCTAC }\end{array}$ & 178 & GGA & NM-0418-000013 & 58 & - \\
\hline RL-LE-030 & $\begin{array}{l}\text { F: CTTGGGAAGGAGGAATGG } \\
\text { R: GTGGGACCAATATGAGGACAGT }\end{array}$ & 164 & TACT & NM-0418-000014 & 59 & - \\
\hline RL-LE-031 & $\begin{array}{l}\text { F: ACTTCAGTTACAGCGACTCTGC } \\
\text { R: GTCGGAGACTGTGCGTTC }\end{array}$ & 194 & CAG & NM-0418-000015 & 58 & PAS domain, PAS domain \\
\hline RL-LE-032 & $\begin{array}{l}\text { F: GTAGAAGGTGCACCAGTTTCTG } \\
\text { R: CGTCTCTTACCAGGAATCACAC }\end{array}$ & 190 & AGG & NM-0418-000016 & 59 & - \\
\hline
\end{tabular}


Table 2. Cont.

\begin{tabular}{|c|c|c|c|c|c|c|}
\hline Marker & Primer Sequences $\left(5^{\prime}-3^{\prime}\right)$ & Expected Size & Motif & GenBank Accession No. & $\mathrm{Ta}\left({ }^{\circ} \mathrm{C}\right)$ & Description \\
\hline RL-LE-033 & $\begin{array}{l}\text { F: GACAGAAGAAGGACTTACCAGC } \\
\text { R: CCAGAGCCCAAGGATAACTT }\end{array}$ & 197 & $\mathrm{CT}$ & NM-0418-000017 & 58 & - \\
\hline RL-LE-034 & $\begin{array}{l}\text { F: AGGTGGAGTTGAGTGTTTGAGG } \\
\text { R: AGTCTCAGGAGACCTTCACTAGC }\end{array}$ & 170 & $\mathrm{TA}$ & NM-0418-000018 & 59 & - \\
\hline RL-LE-035 & $\begin{array}{l}\text { F: GTCGGAAGCTTTATGACACG } \\
\text { R: TCAACTTTCTGCTCCCTCAC }\end{array}$ & 196 & GAG & NM-0418-000019 & 58 & - \\
\hline RL-LE-036 & $\begin{array}{l}\text { F: TCTAGCTCGGTGAGCAATGT } \\
\text { R: GAGACCTTGAGGAAGAGACTCC }\end{array}$ & 181 & CG & NM-0418-000020 & 59 & - \\
\hline RL-LE-037 & $\begin{array}{l}\text { F: CTCTCATCCTTAAGAACCTCCC } \\
\text { R: GAGAAGCTTACATATGGTCCCG }\end{array}$ & 198 & CGA & NM-0418-000021 & 59 & - \\
\hline RL-LE-038 & $\begin{array}{l}\text { F: CGTTTGAGTGTCAACGGTCT } \\
\text { R: CATGTCAGACTAGTCAGGGGTC }\end{array}$ & 199 & $\mathrm{AT}$ & NM-0418-000022 & 59 & - \\
\hline RL-LE-039 & $\begin{array}{l}\text { F: GTACGAGGACAGCAATACAGC } \\
\text { R: GCTTCTATATCTCCTCTGCCCT }\end{array}$ & 200 & GA & NM-0418-000023 & 58 & - \\
\hline RL-LE-040 & $\begin{array}{l}\text { F: GGTTTCCTCTCACACCTTACCT } \\
\text { R: GAAAATGTGCTGTAGCGAGC }\end{array}$ & 178 & $\mathrm{CT}$ & NM-0418-000024 & 59 & - \\
\hline RL-LE-041 & $\begin{array}{l}\text { F: GGTGTATAAAGAGAGCCCTTGG } \\
\text { R: CCCCTTATCCAGTCTACTGCTAC }\end{array}$ & 153 & AG & NM-0418-000025 & 59 & SNF2 family N-terminal domain, Ring finger domain \\
\hline RL-LE-042 & $\begin{array}{l}\text { F: TCCTCTGCTTCACTAAGTCTCC } \\
\text { R: AGTACTCGCAAGGCAGGTAAG }\end{array}$ & 167 & TCG & NM-0418-000026 & 58 & STAG domain \\
\hline RL-LE-043 & $\begin{array}{l}\text { F: GTTCGTCACTCGGTACTTTCC } \\
\text { R: AGATGCAGGAGTATGACCTGAC }\end{array}$ & 177 & $\mathrm{AC}$ & NM-0418-000027 & 58 & - \\
\hline RL-LE-044 & $\begin{array}{l}\text { F: GTAAGCCTAAGGAGGGTGGAG } \\
\text { R: CACCTCCTTCATCTGGTCC }\end{array}$ & 198 & GGA & NM-0418-000028 & 59 & WH1 domain, P21-Rho-binding domain \\
\hline RL-LE-045 & $\begin{array}{l}\text { F: ACATCTGAGAGGTCGTACGCT } \\
\text { R: GTACCGAAGCGAGCAAGTT }\end{array}$ & 164 & CA & NM-0418-000029 & 59 & $\begin{array}{l}\text { Cytochrome b5-like heme/steroid binding domain, } \\
\text { Acyl-CoA dehydrogenase, C-terminal domain }\end{array}$ \\
\hline RL-LE-046 & $\begin{array}{l}\text { F: GCACGCAGTGATGAATAGAGAG } \\
\text { R: ACACTTACGGATTTGGCAGG }\end{array}$ & 154 & AG & NM-0418-000030 & 60 & Cytochrome P450 \\
\hline RL-LE-047 & $\begin{array}{l}\text { F: CTACCACTCGTCACTCCTTAGGT } \\
\text { R: GAAGGAGTGTGAAGCTGAAACC }\end{array}$ & 194 & TC & NM-0418-000031 & 60 & - \\
\hline RL-LE-048 & $\begin{array}{l}\text { F: GTGGTGAAGTTACCGACAGG } \\
\text { R: AGGTGCCCAACTTCTGGT }\end{array}$ & 197 & GC & NM-0418-000032 & 58 & Pectate lyase \\
\hline
\end{tabular}


Table 2. Cont.

\begin{tabular}{|c|c|c|c|c|c|c|}
\hline Marker & Primer Sequences $\left(5^{\prime}-3^{\prime}\right)$ & Expected Size & Motif & GenBank Accession No. & $\mathrm{Ta}\left({ }^{\circ} \mathrm{C}\right)$ & Description \\
\hline RL-LE-049 & $\begin{array}{l}\text { F: GCTACCTAGATCCTCCTAGATCG } \\
\text { R: GACTACGTCAAGTTGAGGATGC }\end{array}$ & 184 & GA & NM-0418-000033 & 58 & - \\
\hline RL-LE-050 & $\begin{array}{l}\text { F: TACCCGAAGGAACTAACGAGTC } \\
\text { R: GTCGTCGTATAACGACTCATCC }\end{array}$ & 200 & TG & NM-0418-000034 & 59 & - \\
\hline RL-LE-051 & $\begin{array}{l}\text { F: ACTCTGCTGCCACTCTTGAC } \\
\text { R: GACCGTCTCTAGCTTCTTGATG }\end{array}$ & 172 & $\mathrm{CT}$ & NM-0418-000035 & 58 & Short chain dehydrogenase \\
\hline RL-LE-052 & $\begin{array}{l}\text { F: CTAAAGCAACGGTAGACGTAGG } \\
\text { R: ACAACAAACGCTAGAGCGAG }\end{array}$ & 178 & GCT & NM-0418-000036 & 58 & - \\
\hline RL-LE-053 & $\begin{array}{l}\text { F: CTCAACGTCTCATTCCCTTC } \\
\text { R: CTCGAGTTGAGGGTGAGGTTAT }\end{array}$ & 179 & GTT & NM-0418-000037 & 58 & - \\
\hline RL-LE-054 & $\begin{array}{l}\text { F: GAATCAGCTAGACCATCTCTGC } \\
\text { R: TCTTTACCCGTCTTGTCTGC }\end{array}$ & 200 & GAT & NM-0418-000038 & 58 & - \\
\hline RL-LE-055 & $\begin{array}{l}\text { F: CTGGGGATAGTGATATCGAGAG } \\
\text { R: GTAAACCCGCTCCTTTGTGT }\end{array}$ & 165 & CTTT & NM-0418-000039 & 58 & - \\
\hline RL-LE-056 & $\begin{array}{l}\text { F: GCGGTCCTGAGTACAAAGTAGT } \\
\text { R: CTACGTACGGAGGAATCTAGTGC }\end{array}$ & 159 & TATC & NM-0418-000040 & 58 & - \\
\hline RL-LE-057 & $\begin{array}{l}\text { F: AGGAGAACGGAACCGAAGTTAC } \\
\text { R: CAGTAGACGTTGCTTACTGCAC }\end{array}$ & 160 & $\mathrm{AT}$ & NM-0418-000041 & 59 & Protein of unknown function DUF262 \\
\hline RL-LE-058 & $\begin{array}{l}\text { F: GTCGTAGAACTTGCACGAGTC } \\
\text { R: GAAGTTCTCCGCTATCCTCTC }\end{array}$ & 163 & GCA & NM-0418-000042 & 57 & - \\
\hline RL-LE-059 & $\begin{array}{l}\text { F: CGGAGATGTACCAATTCCTG } \\
\text { R: GCATTCGCCGTCTATACGAT }\end{array}$ & 193 & TG & NM-0418-000043 & 59 & - \\
\hline RL-LE-060 & $\begin{array}{l}\text { F: ACTCAGCGCACATCTAGCTT } \\
\text { R: CAGGGAGAAGAAAGTCACGA }\end{array}$ & 191 & TCA & NM-0418-000044 & 58 & - \\
\hline
\end{tabular}


These 44 SSR markers were analyzed in 23 cultivars of shiitake mushroom. The major allele frequency $\left(\mathrm{M}_{\mathrm{AF}}\right)$ ranged from 0.13 to 0.94 , with an average of 0.575 . The number of genotypes $\left(\mathrm{N}_{\mathrm{G}}\right)$, ranged from 2 to 11 , with an average of 5.5, and the number of alleles $\left(\mathrm{N}_{\mathrm{A}}\right)$ ranged from 2 to 11 with an average of 4.9 alleles. The observed heterozygosity $\left(\mathrm{H}_{\mathrm{O}}\right)$ ranged from 0.00 to 1.00 , with an average of 0.309 , and the expected heterozygosity $\left(\mathrm{H}_{\mathrm{E}}\right)$, ranged from 0.10 to 0.90 , with an average of 0.552 . The polymorphic information content (PIC) value ranged from 0.10 to 0.89 , with an average of 0.511 . $\mathrm{M}_{\mathrm{AF}}, \mathrm{N}_{\mathrm{G}}, \mathrm{N}_{\mathrm{A}}, \mathrm{H}_{\mathrm{O}}, \mathrm{H}_{\mathrm{E}}$, and PIC per locus had wide ranges among the markers (Table 3).

Table 3. Diversity statistics from each primer used for screening 23 cultivars of shiitake mushroom (Lentinula edodes).

\begin{tabular}{|c|c|c|c|c|c|c|}
\hline Marker & $\mathbf{M}_{\mathrm{AF}}$ & $\mathrm{N}_{\mathrm{G}}$ & $\mathbf{N}_{\mathrm{A}}$ & $\mathrm{H}_{\mathrm{O}}$ & $\mathbf{H}_{\mathrm{E}}$ & PIC \\
\hline RL-LE-017 & 0.13 & 11 & 11 & 0.00 & 0.9 & 0.89 \\
\hline RL-LE-018 & 0.29 & 6 & 6 & 0.08 & 0.77 & 0.74 \\
\hline RL-LE-019 & 0.41 & 7 & 5 & 0.87 & 0.68 & 0.63 \\
\hline RL-LE-020 & 0.67 & 3 & 3 & 0.00 & 0.5 & 0.45 \\
\hline RL-LE-021 & 0.52 & 11 & 10 & 0.43 & 0.68 & 0.66 \\
\hline RL-LE-022 & 0.70 & 5 & 4 & 0.04 & 0.48 & 0.45 \\
\hline RL-LE-023 & 0.63 & 6 & 5 & 0.09 & 0.55 & 0.51 \\
\hline RL-LE-024 & 0.80 & 4 & 4 & 0.13 & 0.33 & 0.31 \\
\hline RL-LE-025 & 0.43 & 7 & 6 & 0.64 & 0.72 & 0.68 \\
\hline RL-LE-026 & 0.34 & 9 & 6 & 0.68 & 0.74 & 0.69 \\
\hline RL-LE-027 & 0.50 & 5 & 5 & 0.26 & 0.64 & 0.58 \\
\hline RL-LE-028 & 0.37 & 6 & 4 & 0.35 & 0.73 & 0.68 \\
\hline RL-LE-029 & 0.81 & 3 & 3 & 0.00 & 0.32 & 0.29 \\
\hline RL-LE-030 & 0.65 & 4 & 3 & 0.26 & 0.51 & 0.46 \\
\hline RL-LE-031 & 0.46 & 4 & 6 & 1.00 & 0.68 & 0.63 \\
\hline RL-LE-032 & 0.87 & 3 & 4 & 0.22 & 0.24 & 0.22 \\
\hline RL-LE-033 & 0.46 & 10 & 9 & 0.48 & 0.72 & 0.69 \\
\hline RL-LE-034 & 0.50 & 6 & 6 & 0.22 & 0.68 & 0.64 \\
\hline RL-LE-035 & 0.65 & 5 & 5 & 0.17 & 0.52 & 0.47 \\
\hline RL-LE-036 & 0.72 & 3 & 3 & 0.04 & 0.42 & 0.35 \\
\hline RL-LE-037 & 0.50 & 6 & 4 & 0.39 & 0.62 & 0.55 \\
\hline RL-LE-038 & 0.39 & 7 & 9 & 1.00 & 0.72 & 0.68 \\
\hline RL-LE-039 & 0.50 & 3 & 3 & 0.13 & 0.56 & 0.46 \\
\hline RL-LE-040 & 0.52 & 6 & 5 & 0.52 & 0.66 & 0.61 \\
\hline RL-LE-041 & 0.43 & 4 & 5 & 0.09 & 0.65 & 0.58 \\
\hline RL-LE-042 & 0.80 & 4 & 3 & 0.04 & 0.33 & 0.3 \\
\hline RL-LE-043 & 0.87 & 2 & 2 & 0.00 & 0.23 & 0.2 \\
\hline RL-LE-044 & 0.76 & 3 & 3 & 0.42 & 0.37 & 0.32 \\
\hline RL-LE-045 & 0.39 & 6 & 5 & 0.35 & 0.73 & 0.69 \\
\hline RL-LE-046 & 0.43 & 7 & 6 & 0.09 & 0.71 & 0.66 \\
\hline RL-LE-047 & 0.48 & 10 & 6 & 0.35 & 0.69 & 0.65 \\
\hline RL-LE-048 & 0.43 & 8 & 5 & 0.39 & 0.7 & 0.66 \\
\hline RL-LE-049 & 0.41 & 4 & 5 & 1.00 & 0.66 & 0.6 \\
\hline RL-LE-050 & 0.94 & 2 & 2 & 0.00 & 0.1 & 0.1 \\
\hline RL-LE-051 & 0.28 & 9 & 6 & 0.65 & 0.79 & 0.76 \\
\hline RL-LE-052 & 0.70 & 5 & 5 & 0.09 & 0.47 & 0.43 \\
\hline RL-LE-053 & 0.39 & 10 & 10 & 0.61 & 0.79 & 0.77 \\
\hline RL-LE-054 & 0.76 & 5 & 3 & 0.09 & 0.39 & 0.36 \\
\hline RL-LE-055 & 0.72 & 4 & 4 & 0.13 & 0.44 & 0.38 \\
\hline RL-LE-056 & 0.59 & 5 & 4 & 0.68 & 0.58 & 0.53 \\
\hline RL-LE-057 & 0.87 & 3 & 3 & 0.09 & 0.23 & 0.22 \\
\hline RL-LE-058 & 0.93 & 3 & 3 & 0.04 & 0.12 & 0.12 \\
\hline RL-LE-059 & 0.35 & 6 & 5 & 0.30 & 0.74 & 0.69 \\
\hline RL-LE-060 & 0.91 & 2 & 2 & 0.17 & 0.16 & 0.15 \\
\hline Mean & 0.575 & 5.5 & 4.9 & 0.309 & 0.552 & 0.511 \\
\hline
\end{tabular}

$\left(\mathrm{M}_{\mathrm{AF}}\right)$, Major allele frequency; $\left(\mathrm{N}_{\mathrm{G}}\right)$, number of genotypes; $\left(\mathrm{N}_{\mathrm{A}}\right)$, number of alleles; $\left(\mathrm{H}_{\mathrm{O}}\right)$, observed heterozygosity; $\left(\mathrm{H}_{\mathrm{E}}\right)$, expected heterozygosity; and (PIC), polymorphic information content. 
SSR marker information for the determination of cultivars in other cultivated mushrooms have been released. In the SSR markers developed for the determination of black wood ear (Auricularia auricular-judae) cultivars, the PIC value ranged from 0.10 to 0.84 , with an average of 0.47 , and $\mathrm{N}_{\mathrm{A}}$ ranged from 2-11, with an average of 4.7 (using 17 SSR markers and 16 cultivars) [25]. In the white button mushroom (Agaricus bisporus), the allele frequency ranged from 0.02-0.94, with an average of 0.18 , and $\mathrm{H}_{\mathrm{O}}$ ranged from 0.00 to 0.83 , with an average of 0.35 (using 33 SSR markers, 6 cultivars and 17 wild types of $A$. bisporus, and 2 wild types of $A$. bisporus var. burnettii) [26]. In the golden needle mushroom (F. velutipes), the PIC value ranged from 0.13 to 0.69 , with an average of 0.42 (using 55 SSR markers and 14 cultivars) [27]. In the oyster mushroom (P. ostreatus), the average of $\mathrm{N}_{\mathrm{A}}$ was approximately 4.7. $\mathrm{H}_{\mathrm{O}}$ ranged from 0.027 to 0.946 , with an average of 0.398 , and $\mathrm{H}_{\mathrm{E}}$ ranged from 0.027 to 0.810 , with an average of 0.549 (using 36 SSR markers, and 37 cultivars including P. sajor-caju, 1 P. eryngii, 1 P. cornucopiae var. citrinopileatus, and 1 P. nebrodensis) [28]. The SSR markers developed in this study showed similar diversity values with the SSR markers of other edible mushrooms. The 20 markers with PIC values above 0.6 in the 44 newly developed markers are useful for identification among strains or cultivars of shiitake mushroom. The SSR markers developed in this study were able to distinguish 23 shiitake mushroom strains, which are broadly cultivated in Korea (Supplementary Materials Figure S2).

\section{Conclusions}

We have developed 44 novel SSR markers for the determination of shiitake mushroom cultivars. SSR marker development was performed based on NGS-based genome sequencing data. The efficacy and availability of the developed SSR markers were evaluated by application to distinguishing 23 shiitake mushroom cultivars. These new markers can be used for molecular breeding, cultivar determination, genetic structure research, and further applications in cultivated and wild types of shiitake mushrooms.

Supplementary Materials: The following are available online at www.mdpi.com/2073-4425/8/4/109/s1, Figure S1: The development process of SSR marker developed using genome sequencing for Lentinula edodes, Figure S2: Distinguished Lentinula edodes strains using the 44 novel SSR markers developed in this study.

Acknowledgments: This study was performed with the support of the "Golden Seed Project (Center for Horticultural Seed Development, No. 213007-05-1-SBH20)".

Author Contributions: Hwa-Yong Lee, Suyun Moon, and Donghwan Shim contributed equally to this work, analyzed the data, and wrote the paper; Chang Pyo Hong and Yi Lee performed the experiments; and Chang-Duck Koo, Jong-Wook Chung, and Hojin Ryu designed the experiments. All authors read and approved the manuscript.

Conflicts of Interest: The authors declare no conflict of interest.

\section{References}

1. International Mycological Association. Mycobank Database Fungal Databases, Nomenclature and Species Banks. Available online: http:/ / www.mycobank.org/ (accessed on 29 September 2016).

2. Bak, W.C.; Park, Y.A.; Park, J.H. KFRI Forest Policy Issue: Present Situation and Future of Oak Mushroom Industry; Korea Forest Research Institute: Seoul, Korea, 2013; p. 17.

3. Kim, K.H.; Ka, K.H.; Kang, J.H.; Kim, S.; Lee, J.W.; Jeon, B.K.; Yun, J.K.; Park, S.R.; Lee, H.J. Identification of Single Nucleotide Polymorphism Markers in the Laccase Gene of Shiitake Mushrooms (Lentinula edodes). Mycobiology 2015, 43, 75-80. [CrossRef] [PubMed]

4. Royse, D.J. A global perspective on the high five: Agaricus, Pleurotus, Lentinula, Auricularia \& Flammulina. In Proceedings of the 8th International Conference on Mushroom Biology and Mushroom Products, New Delhi, India, 19-22 November 2014.

5. Chihara, G.; Hamuro, J.; Maeda, Y.; Arai, Y.; Fukuoka, F. Fractionation and Purification of the Polysaccharides with Marked Antitumor Activity, Especially Lentinan, from Lentinus edodes (Berk.) Sing, (an Edible Mushroom). Cancer Res. 1970, 30, 2776-2781. [PubMed] 
6. Bisen, P.S.; Baghel, R.K.; Sanodiya, B.S.; Thakur, G.S.; Prasad, G.B. Lentinus edodes: A macrofungus with pharmacological activities. Curr. Med. Chem. 2010, 17, 2419-2430. [CrossRef] [PubMed]

7. Chakravarty, B. Trends in mushroom cultivation and breeding. Aust. J. Agric. Eng. 2011, 2, 102-109.

8. Forest Mushroom Research Center. Cultivation Technique of Oak Mushroom; Forest Mushroom Research Center: Yeoju, Korea, 2015; pp. 58-60.

9. Sonnenberg, A.S.M.; Johan, J.P.B.; Patrick, M.H.; Brian, L.; Wei, G.; Amrah, W.; Jurriaan, J.M. Breeding and strain protection in the button mushroom Agaricus bisporus. In Proceedings of the 7th International Conference on Mushroom Biology and Mushroom Products, Arcachon, France, 4-7 October 2011; pp. 7-15.

10. Kulkarni, R.K. DNA Polymorphisms in Lentinula edodes, the Shiitake Mushroom. App. Environ. Microbiol. 1991, 57, 1735-1739.

11. Zhang, Y.; Molina, F.I. Strain typing of Lentinula edodes by random amplified polymorphic DNA assay. FEMS Microbiol. Lett. 1995, 131, 17-20. [CrossRef] [PubMed]

12. Fu, L.Z.; Zhang, H.Y.; Wu, X.Q.; Li, H.B.; Wei, H.L.; Wu, Q.Q.; Wang, L.A. Evaluation of genetic diversity in Lentinula edodes strains using RAPD, ISSR and SRAP markers. World J. Microbl. Biotech. 2010, 26, 709-716. [CrossRef]

13. Wu, X.; Li, H.; Zhao, W.; Fu, L.; Peng, H.; He, L.; Cheng, J. SCAR makers and multiplex PCR-based rapid molecular typing of Lentinula edodes strains. Curr. Microbiol. 2010, 61, 381-389. [CrossRef] [PubMed]

14. Terashima, K.; Matsumoto, T. Strain typing of shiitake (Lentinula edodes) cultivars by AFLP analysis, focusing on a heat-dried fruiting body. Mycoscience 2004, 45, 79-82. [CrossRef]

15. Zhang, R.; Huang, C.; Zheng, S.; Zhang, J.; Ng, T.B.; Jiang, R.; Zuo, X.; Wang, H. Strain-typing of Lentinula edodes in China with inter simple sequence repeat markers. Appl. Microbiol. Biotechnol. 2007, 74, 140-145. [CrossRef] [PubMed]

16. Liu, J.; Wang, Z.R.; Li, C.; Bian, Y.B.; Xiao, Y. Evaluating genetic diversity and constructing core collections of Chinese Lentinula edodes cultivars using ISSR and SRAP markers. J. Basic Microbiol. 2015, 55, 749-760. [CrossRef] [PubMed]

17. Li, H.B.; Wu, X.Q.; Peng, H.Z.; Fu, L.Z.; Wei, H.L.; Wu, Q.Q.; Jin, Q.Y.; Li, N. New available SCAR markers: Potentially useful in distinguishing a commercial strain of the superior type from other strains of Lentinula edodes in China. Appl. Microbiol. Biotechnol. 2008, 81, 303-309. [CrossRef] [PubMed]

18. Liu, J.Y.; Ying, Z.H.; Liu, F.; Liu, X.R.; Xie, B.G. Evaluation of the use of SCAR markers for screening genetic diversity of Lentinula edodes strains. Curr. Microbiol. 2012, 64, 317-325. [CrossRef] [PubMed]

19. Miah, G.; Rafii, M.Y.; Ismail, M.R.; Puteh, A.B.; Rahim, H.A.; Islam, Kh.N.; Latif, M.A. A review of microsatellite markers and their applications in rice breeding programs to improve blast disease resistance. Int. J. Mol. Sci. 2013, 14, 22499-22528. [CrossRef] [PubMed]

20. Xiao, Y.; Liu, W.; Dai, Y.; Fu, C.; Bian, Y. Using SSR markers to evaluate the genetic diversity of Lentinula edodes' natural germplasm in China. World J. Microbiol. Biotechnol. 2010, 26, 527-536. [CrossRef]

21. Zalapa, J.E.; Cuevas, H.; Zhu, H.; Steffan, S.; Senalik, D.; Zeldin, E.; McCown, B.; Harbut, R.; Simon, P. Using next-generation sequencing approaches to isolate simple sequence repeat (SSR) loci in the plant sciences. Am. J. Bot. 2012, 99, 193-208. [CrossRef] [PubMed]

22. Ekblom, R.; Galindo, J. Applications of next generation sequencing in molecular ecology of non-model organisms. Heredity 2011, 107, 1-15. [CrossRef] [PubMed]

23. Shim, D.; Park, S.G.; Kim, K.; Bae, W.; Lee, G.W.; Ha, B.S.; Ro, H.S.; Kim, M.; Ryoo, R.; Rhee, S.K.; et al. Whole genome de novo sequencing and genome annotation of the world popular cultivated edible mushroom, Lentinula edodes. J. Biotechnol. 2016, 223, 24-25. [CrossRef] [PubMed]

24. Liu, K.; Muse, S.V. PowerMarker: A intergrated analysis environment for genetic marker analysis. Bioinformatics 2005, 21, 2128-2129. [CrossRef] [PubMed]

25. Zhang, R.Y.; Hu, D.D.; Gu, J.G.; Hi, Q.X.; Zuo, X.M.; Wnag, H.X. Development of SSR markers for typing cultivars in the mushroom Auricularia auricula-judae. Mycol. Prog. 2012, 11, 578-592. [CrossRef]

26. Foulongne-Oriol, M.; Spataro, M.; Savoie, J.M. Novel microsatellite markers suitable for genetic studies in the white button mushroom Agaricus bisporus. Appl. Microbiol. Biotechnol. 2009, 84, 1125-1135. [CrossRef] [PubMed] 
27. Zhang, R.; Hu, D.; Zhang, J.; Zuo, X.; Jiang, R.; Wang, H.; Ng, T.B. Development and characterization of simple sequence repeat (SSR) markers for the mushroom Flammulina velutipes. J. Biosci. Bioeng. 2010, 110, 273-275. [CrossRef] [PubMed]

28. Ma, K.H.; Lee, G.A.; Lee, S.Y.; Gwag, J.G.; Kim, T.S.; Kong, W.S.; Seo, K.I.; Lee, G.S.; Park, Y.J. Development and characterization of new microsatellite markers for the oyster mushroom (Pleurotus ostreatus). J. Microbiol. Biotechnol. 2009, 19, 851-857. [CrossRef] [PubMed]

(C) 2017 by the authors. Licensee MDPI, Basel, Switzerland. This article is an open access article distributed under the terms and conditions of the Creative Commons Attribution (CC BY) license (http:/ / creativecommons.org/licenses/by/4.0/). 